\title{
Lung immunity: necessity is the mother of induction
}

\author{
Max Corbett \& Jean-Pierre Kraehenbuhl
Lymphoid structures, complete with B cell follicles, germinal centers, and T cell zones, form in the lung in response to influenza infection. This finding casts new light on respiratory immunity, showing that it is more self-contained than previously thought (927-934).

Aside from structures within the nasal cavity, all secondary lymphoid organs, such as spleen, lymph nodes and Peyer's patches, are present and functional in mice just after birth $^{1}$. In this issue, Moyron-Quiroz et al. ${ }^{2}$ reveal an additional facet of lymphoid organogenesis, showing that adult mice, upon influenza infection, form lymphoid structures de novo in the lung. What is more, these structures are capable of mounting delayed yet competent adaptive immune responses, which are able to contain the pulmonary infection. The study should spur an intense search for the signals that prompt formation of such structures, which form independently of the signal required for classical lymph node development.

The researchers laid the groundwork for this study in a previous study, where they examined immune responses to influenza in lymphotoxin- $\alpha$ (LT $\alpha)$ knockout mice ${ }^{3}$. LT $\alpha$, a member of the TNF family of cytokines, is a potent modulator of the immune response $\mathrm{e}^{4}$. Additionally, LT $\alpha$ is required for the induction of secondary lymphoid organs during embryogenesis. The LT $\alpha$ knockout mice entirely lack lymph nodes and Peyer's patches but have spleens and nasal-associated lymphoid tissues. However, these two latter structures have disrupted architectures lacking follicular dendritic cells, a nonbone-marrow-derived stromal cell type that coordinates the selection of antigen-specific B cells ${ }^{1}$. Not surprisingly, the LT $\alpha$ knockout mice show defective isotype switching and affinity maturation of antibodies, as well as severely impaired cellular responses to systemic infections $s^{5-8}$. It was therefore unexpected when Lund et al. showed that pulmonary influenza infection leads to high titers of flu-specific IgM, IgG and IgA antibodies and similar numbers of flu-specific

The authors are at the Swiss Institute for Experimental Cancer Research (ISREC), 1066 Epalinges, Switzerland, and the Division of Immunology and Allergy, Centre Hospitalier Universitaire Vaudois (CHUV), 1011 Lausanne, Switzerland.

e-mail: max.corbett@isrec.unil.ch

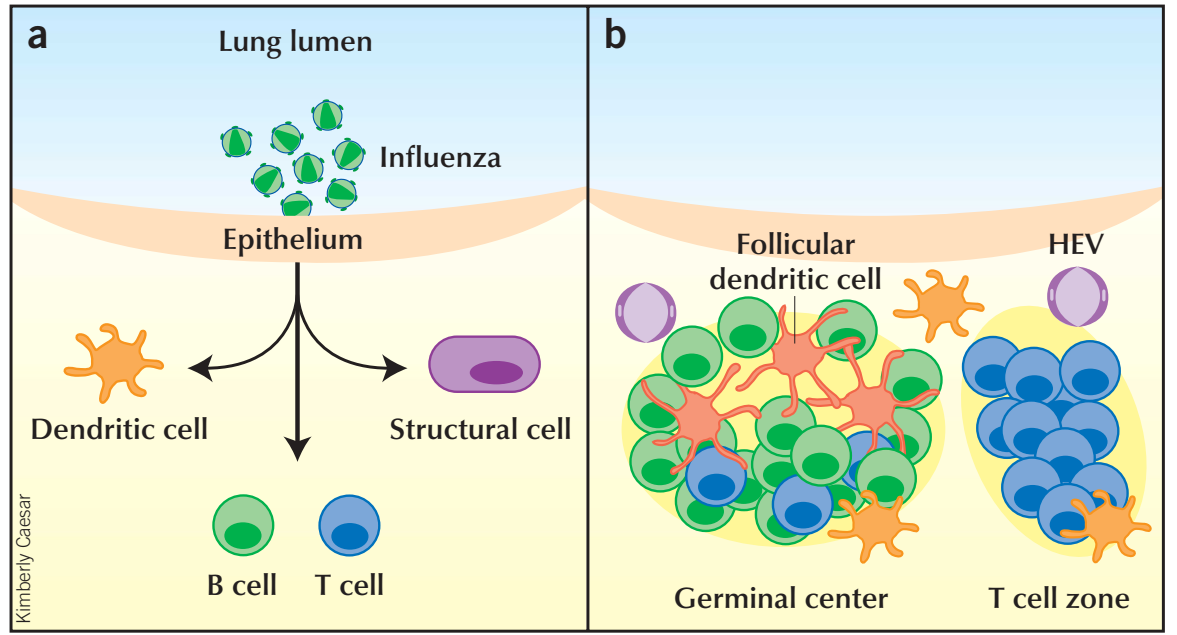

Figure 1 Induced secondary lymphoid tissue formation in the lung upon influenza infection. (a) Pulmonary infection generates bronchus-associated lymphoid tissue (BALT) de novo, independently of LT $\alpha$. Undefined mediators, possibly originating from the epithelium, act on resident lung cells, such as macrophages, dendritic cells, lymphocytes and structural cells (fibroblasts, endothelial and mesenchymal cells), to coordinate BALT formation. (b) Once formed, BALT sustains adaptive immune responses. Entering the BALT through high endothelial venules (HEV) expressing PNAd and the lymphocyte attractant CCL21, naive B and T lymphocytes encounter their cognate antigens and segregate into distinct $B$ and T cell rich zones. Within these structures, proliferating CD4, CD8 and $B$ cells are observed. Once activated and clonally expanded, lymphocytes exit the BALT through efferent lymphatics.

CD8 T cells in the LT $\alpha$ knockout mice as in normal wild-type animals ${ }^{3}$. What is the origin of this competent, pulmonary-induced, adaptive immune response in these mice?

Moyron-Quiroz et al. ${ }^{2}$ reveal the answer, demonstrating that the flu-specific immune responses originate from the bronchus-associated lymphoid tissue (BALT) within the lung itself. BALT is a submucosal lymphoid aggregate found beneath the epithelium at bronchi branches within the lower respiratory tract $^{9}$ (Fig. 1). Unlike classical lymph nodes, BALT is not encapsulated but rather integrated directly in the lung tissue. This lymphoid structure does not draw upon afferent lymphatics for antigen retrievalinstead, antigen is sampled directly from the lung lumen through specialized epithelial cells ${ }^{10}$. The presence of BALT varies among vertebrate species as well as with the age of the animal. External factors such as high antigen load, infection and inflammation seem to promote BALT formation.
In the current study, the authors found that induced BALT structures (iBALT) were formed independently of LT $\alpha$ expression. Ten days after influenza infection, both wildtype and LT $\alpha$ knockout mice showed iBALT, as evidenced by the presence of prominent $B$ cell-containing structures. The lymphoid aggregates, however, were not identical in the two mouse strains. Despite expression in both mouse strains of the chemokines CXCL13 and CCL21-B and B/T cell attractants, respectively - the iBALT in influenzachallenged LT $\alpha$ knockout mice did not have distinct $\mathrm{B}$ and $\mathrm{T}$ zones. The iBALT was, however, still able to generate flu-specific CD8 and isotype-switched antibody responses, the latter in a fashion seemingly independent of follicular dendritic cells.

To investigate the requirements of the lung-induced adaptive immune responses, Moyron-Quiroz et al. ${ }^{2}$ generated a series of chimeric mice. In LT $\alpha$ knockout mice reconstituted with wild-type bone mar- 
row-which lacked spleens, lymph nodes, and Peyer's patches-the iBALT contained all of the classical immune cells of a lymph node that support an effective immune response. These included lymphocytes and follicular dendritic cells. Moreover, B cell follicles, germinal centers, and $\mathrm{T}$ cell-rich zones were present in the iBALT, with actively proliferating lymphocytes observed in the latter two structures.

Consistent with this classical lymphoid architecture was the presence of CXCL13 and CCL21, respectively in, and around, germinal centers. These molecules no doubt orchestrate the appropriate movements of the lymphoid cells. Migration of the lymphocytes from the blood was possibly mediated in part by the selectin ligand peripheral lymph node addressin (PNAd), along with CCL21, as both were expressed on endothelial cells within the iBALT. As compared with the LT $\alpha$ knockout mice, the $\mathrm{Lta}^{-/}$/wild-type chimeras mounted swifter adaptive immune responses that more effectively controlled the influenza infection. This was no doubt due to the organized structure of the iBALT. Thus, although iBALT is formed in an LT $\alpha$ autonomous manner, the efficiency of iBALT-induced immunity is LT $\alpha$ dependent.

Collectively, these findings suggest a LT $\alpha$ independent mechanism of iBALT induction. So where is the BALT-initiating signal coming from? One distinct possibility is the infected epithelium itself. Long thought to be merely physical barriers to the outside world, mucosal epithelia are now being recognized as playing a pivotal role in innate immunity. Grace pathogen-associated molecular pattern-recognition receptors, such as the Tolllike receptor (TLR) family, on epithelial cells detect invading microbes and set immune responses in motion ${ }^{11}$. For example, in response to bacterial components, such as flagellin, gut epithelial cells release chemokines that recruit immune cells, particularly dendritic cells, to the epithelium ${ }^{12}$. In fact alveolar epithelial cells have recently been shown to express TLR2, TLR4 and TLR5 (refs. 13,14). In addition to chemokines, stimulated epithelial cells express a variety of cytokines and growth factors $^{15}$. Thus, it is imaginable that within the lung tissues these factors act on resident cells, such as macrophages, fibroblasts, mesenchymal cells, endothelial cells and lymphocytes, which in turn coordinate their formation into BALT structures. Supporting this notion of an epithelium-derived impetus for iBALT formation is the presence of specialized sampling epithelial cells in the lung mucosa even before BALT formation ${ }^{10}$.

Moyron-Quiroz et al. ${ }^{2}$ have documented the formation of inducible pulmonary secondary lymphoid tissues, which are competent to mount $\mathrm{B}$ and $\mathrm{T}$ adaptive immune responses. Further investigation of this phe- nomenon is merited, especially in respect to vaccine development. By understanding the mechanism behind iBALT formation, vaccine formulations or pretreatment for aerosol delivery of immunogens can be envisioned. Such procedures would boost pulmonary responses to lung specific pathogens such as influenza and severe acute respiratory syndrome (SARS).

1. Mebius, R.E. Nat. Rev. Immunol. 3, 292-303 (2003).

2. Moyron-Quiroz, J.E. et al. Nat. Med. 10, 927-934 (2004).

3. Lund, F.E. et al. J. Immunol. 169, 5236-5243 (2002).

4. Pfeffer, K. Cytokine Growth Factor Rev. 14, 185-191 (2003)

5. Fu, Y.X. et al. J. Exp. Med. 185, 2111-2120 (1997).

6. Banks, T.A. et al. J. Immunol. 155, 1685-1693 (1995).

7. Kumaraguru, U., Davis, I.A., Deshpande, S., Tevethia, S.S. \& Rouse, B.T. J. Immunol. 166 1066-1074 (2001).

8. Suresh, M. et al. J. Virol. 76, 3943-3951 (2002).

9. Sminia, T., van der Brugge-Gamelkoorn, G.J. \& Jeurissen, S.H. Crit. Rev. Immunol. 9, 119-150 (1989)

10. Tango, M., Suzuki, E., Gejyo, F. \& Ushiki, T. Arch. Histol. Cytol. 63, 81-89 (2000).

11. Neutra, M.R., Mantis, N.J. \& Kraehenbuhl, J.P. Nat. Immunol. 2, 1004-1009 (2001).

12. Sierro, F. et al. Proc. Natl. Acad. Sci. USA 98, 13722-13727 (2001).

13. Armstrong, L. et al. Am. J. Respir. Cell Mol. Biol. 31, 241-245 (2004).

14. Adamo, R., Sokol, S., Soong, G., Gomez, M.I. \& Prince, A. Am. J. Respir. Cell Mol. Biol. 30 627-634 (2004).

15. Salvi, S. \& Holgate, S.T. Clin. Exp. Allergy 29, 1597-1605 (1999).

\section{Maiming mitochondria in familial ALS}

\section{Valina L Dawson}

\section{A population of neurons in patients with familial amyotrophic lateral sclerosis have multiple abnormalities, including protein aggregation and mitochondrial dysfunction. Two studies now reveal interactions between these two defects.}

Something is not quite right about the mitochondria of patients with familial amyotrophic lateral sclerosis (ALS). Vacuolation and degeneration of mitochondria occur early in this degenerative disease, which afflicts 6 out of 100,000 individuals. Moreover, deficits in respiration occur in cells expressing a mutant form of the gene encoding $\mathrm{Cu} / \mathrm{Zn}$ superoxide dismutase (SOD-1),

Valina L. Dawson is at the Institute for Cell Engineering and in the Departments of Neurology, Neuroscience and Physiology, John Hopkins University School of Medicine, Baltimore Maryland, 21205, USA.

e-mail:vdawson@jhmi.edu associated with familial cases of ALS. A challenge for the field is to define the relationships among the mitochondrial defects and the many other cellular abnormalities, such as protein aggregates, that may lead to the selective degeneration of motor neurons that occurs in ALS ${ }^{1,2}$.

Two studies in a recent issue of Neuron ${ }^{3,4}$ imply that there is a causal link between SOD-1 mutations, impairment of mitochondrial function and motor neuron degeneration. The studies describe interactions between mutant SOD-1 protein and mitochondria that potentially compromise mitochondrial function and promote apoptosis through release of mitochondrial enzymes. Perhaps more importantly, the work shows that mutant SOD-1 accumulates in the intermembrane space of spinal cord mitochondria, presenting numerous possibilities for ALS pathogenesis. How all this fits together with other aspects of the disorder, and exactly how SOD-1-mitochondrial interactions could promote cell death, remains to be seen.

About $10 \%$ of ALS cases are familial-and about $25 \%$ of these are linked to mutations in the gene encoding SOD-1, which catalyzes the conversion of superoxide anion to hydrogen peroxide ${ }^{2,5}$. SOD-1 is expressed throughout the body, which presents a puzzle, as familial ALS is a disease of selective degeneration of motor neurons and neurons in the motor cortex. 